\title{
FIXED POINT THEOREMS FOR GENERALIZED LIPSCHITZIAN SEMIGROUPS
}

\author{
JONG SOO JUNG and BALWANT SINGH THAKUR
}

(Received 10 March 2000)

\begin{abstract}
Let $K$ be a nonempty subset of a $p$-uniformly convex Banach space $E, G$ a left reversible semitopological semigroup, and $\mathscr{S}=\left\{T_{t}: t \in G\right\}$ a generalized Lipschitzian semigroup of $K$ into itself, that is, for $s \in G,\left\|T_{s} x-T_{s} y\right\| \leq a_{s}\|x-y\|+b_{s}\left(\left\|x-T_{s} x\right\|+\right.$ $\left.\left\|y-T_{s} y\right\|\right)+c_{s}\left(\left\|x-T_{s} y\right\|+\left\|y-T_{s} x\right\|\right)$, for $x, y \in K$ where $a_{s}, b_{s}, c_{s}>0$ such that there exists a $t_{1} \in G$ such that $b_{s}+c_{s}<1$ for all $s \geq t_{1}$. It is proved that if there exists a closed subset $C$ of $K$ such that $\bigcap_{s} \overline{\operatorname{co}}\left\{T_{t} x: t \succeq s\right\} \subset C$ for all $x \in K$, then $\mathscr{S}$ with $\left[(\alpha+\beta)^{p}\left(\alpha^{p}\right.\right.$. $\left.\left.2^{p-1}-1\right) /\left(c_{p}-2^{p-1} \beta^{p}\right) \cdot N^{p}\right]^{1 / p}<1$ has a common fixed point, where $\alpha=\limsup _{s}\left(a_{s}+\right.$ $\left.b_{S}+c_{S}\right) /\left(1-b_{S}-c_{S}\right)$ and $\beta=\limsup _{s}\left(2 b_{s}+2 c_{S}\right) /\left(1-b_{s}-c_{S}\right)$.
\end{abstract}

2000 Mathematics Subject Classification. 47H10.

1. Introduction. Let $K$ be a nonempty subset of a Banach space $E$ and $T$ a mapping of $K$ into itself. The mapping $T$ is said to be Lipschitzian mapping if for each $n \geq 1$, there exists a positive real number $k_{n}$ such that

$$
\left\|T^{n} x-T^{n} y\right\| \leq k_{n}\|x-y\|
$$

for all $x, y$ in $K$. A Lipschitzian mapping is said to be nonexpansive if $k_{n}=1$ for all $n \geq 1$, uniformly $k$-Lipschitzian if $k_{n}=k$ for all $n \geq 1$, and asymptotically nonexpansive if $\lim _{n} k_{n}=1$, respectively. These mappings were first studied by Goebel and Kirk [7] and Goebel, Kirk, and Thele [9]. Lifšic [13] proved that in a Hilbert space a uniformly $k$-Lipschitzian mapping with $k<\sqrt{2}$ has a fixed point. Downing and Ray [4] and Ishihara and Takahashi [12] proved that in a Hilbert space a uniformly $k$-Lipschitzian semigroup with $k<\sqrt{2}$ has a common fixed point. Casini and Maluta [3] and Ishihara and Takahashi [11] proved that a uniformly $k$-Lipschitzian semigroup in a Banach space $E$ has a common fixed point if $k<\sqrt{N(E)}$, where $N(E)$ is the constant of uniformly normal structure.

In these results, the domains of semigroups were assumed to be closed and convex. Ishihara [10] gave fixed point theorems for Lipschitzian semigroups in both Banach and Hilbert spaces in which closedness and convexity of domain were not needed.

Now we consider the following class of mappings, which we call generalized Lipschitzian mapping, whose $n$th iterate $T^{n}$ satisfying the following condition:

$$
\begin{aligned}
\left\|T^{n} x-T^{n} y\right\| \leq & a_{n}\|x-y\|+b_{n}\left(\left\|x-T^{n} x\right\|+\left\|y-T^{n} y\right\|\right) \\
& +c_{n}\left(\left\|x-T^{n} y\right\|+\left\|y-T^{n} x\right\|\right),
\end{aligned}
$$

for each $x, y \in K$ and $n \geq 1$, where $a_{n}, b_{n}, c_{n}$ are the nonnegative constants such that there exists an integer $n_{0}$ such that $b_{n}+c_{n}<1$ for all $n \geq n_{0}$. 
This class of generalized Lipschitzian mappings is more general than the classes of nonexpansive, asymptotically nonexpansive, Lipschitzian and uniformly $k$-Lipschitzian mappings. The above facts can be seen by taking $b_{n}=c_{n}=0$.

In this paper, we prove a fixed point theorem for generalized Lipschitzian semigroups in a $p$-uniformly convex Banach space. Next we give its corollaries in a Hilbert space, in $L^{p}$ spaces, in Hardy space $H^{p}$ and in Sobolev spaces $H^{k, p}$, for $1<p<\infty$ and $k \geq 0$. Our results improve and extend results from $[10,11,12]$.

2. Preliminaries. Let $G$ be a semitopological semigroup, that is, $G$ is a semigroup with a Hausdorff topology such that for each $a \in G$ the mapping $s \rightarrow a \cdot s$ and $s \rightarrow$ $s \cdot a$ from $G$ to $G$ are continuous. A semitopological semigroup $G$ is left reversible if any two closed right ideals of $G$ have nonempty intersection. In this case, $(G, \preceq)$ is a directed system when the binary relation " $\preceq$ ” on $G$ is defined by $a \preceq b$ if and only if $\{a\} \cup \overline{a G} \supseteq\{b\} \cup \overline{b G}$. Examples of left reversible semigroups include commutative and all left amenable semigroups.

Let $K$ be a mapping subset of a Banach space $E$. Then a family $\mathscr{S}=\left\{T_{t}: t \in G\right\}$ of mappings from $K$ into itself is said to be a generalized Lipschitzian semigroup on $K$ if $\mathscr{G}$ satisfies the following:

(i) $T_{t s}(x)=T_{t} T_{s}(x)$ for $t, s \in G$ and $x \in K$;

(ii) the mapping $(s, x) \rightarrow T_{\mathcal{S}}(x)$ from $G \times K$ into $K$ is continuous when $G \times K$ has the product topology;

(iii) for each $s \in G$

$$
\begin{aligned}
\left\|T_{s} x-T_{s} y\right\| \leq & a_{s}\|x-y\|+b_{s}\left(\left\|x-T_{s} x\right\|+\left\|y-T_{s} y\right\|\right) \\
& +c_{s}\left(\left\|x-T_{s} y\right\|+\left\|y-T_{s} x\right\|\right),
\end{aligned}
$$

for $x, y \in K$ where $a_{s}, b_{s}, c_{s}>0$ such that there exists a $t_{1} \in G$ such that $b_{s}+c_{s}<1$ for all $s \geq t_{1}$.

Let $\left\{B_{\alpha}: \alpha \in \wedge\right\}$ be a decreasing net of bounded subsets of a Banach space $E$. For a nonempty subset $K$ of $E$, define

$$
\begin{aligned}
& r\left(\left\{B_{\alpha}\right\}, x\right)=\inf _{\alpha} \sup \left\{\|x-y\|: y \in B_{\alpha}\right\} \\
& r\left(\left\{B_{\alpha}\right\}, K\right)=\inf \left\{r\left(\left\{B_{\alpha}\right\}, x\right): x \in K\right\} \\
& A\left(\left\{B_{\alpha}\right\}, K\right)=\left\{x \in K: r\left(\left\{B_{\alpha}\right\}, x\right)=r\left(\left\{B_{\alpha}\right\}, K\right)\right\} .
\end{aligned}
$$

We know that $r\left(\left\{B_{\alpha}\right\}, \cdot\right)$ is a continuous convex function on $E$ which satisfies the following:

$$
\left|r\left(\left\{B_{\alpha}\right\}, x\right)-r\left(\left\{B_{\alpha}\right\}, y\right)\right| \leq\|x-y\| \leq r\left(\left\{B_{\alpha}\right\}, x\right)+r\left(\left\{B_{\alpha}\right\}, y\right)
$$

for each $x, y \in E$. It is easy to see that if $E$ is reflexive and $K$ is closed convex, then $A\left(\left\{B_{\alpha}\right\}, K\right)$ is nonempty, and moreover, if $E$ is uniformly convex, then it consists of a single point (cf. [14]).

Let $p>1$, and denote by $\lambda$ the number in $[0,1]$ and by $W_{p}(\lambda)$ the function $\lambda \cdot(1-$ $\lambda)^{p}+\lambda^{p} \cdot(1-\lambda)$. 
The functional $\|\cdot\|^{p}$ is said to be uniformly convex (cf. Zălinescu [25]) on the Banach space $E$ if there exists a positive constant $c_{p}$ such that for all $\lambda \in[0,1]$ and $x, y \in E$ the following inequality holds:

$$
\|\lambda x+(1-\lambda) y\|^{p} \leq \lambda\|x\|^{p}+(1-\lambda)\|y\|^{p}-W_{p}(\lambda) \cdot c_{p} \cdot\|x-y\|^{p} .
$$

$\mathrm{Xu}$ [24] proved that the functional $\|\cdot\|^{p}$ is uniformly convex on the whole Banach space $E$ if and only if $E$ is $p$-uniformly convex, that is, there exists a constant $c>0$ such that the moduli of convexity (see [8]) $\delta_{E}(\varepsilon) \geq c \cdot \varepsilon^{p}$ for all $0 \leq \varepsilon \leq 2$.

The normal structure coefficient $N(E)$ of $E$ (cf. [2]) is defined by

$$
\begin{array}{r}
N(E)=\inf \left\{\frac{\operatorname{diam} K}{r_{K}(K)}: K \text { is a bounded convex subset of } E\right. \\
\text { consisting of more than one point }\},
\end{array}
$$

where $\operatorname{diam} K=\sup \{\|x-y\|: x, y \in K\}$ is the diameter of $K$ and $r_{K}(K)=$ $\inf _{x \in K}\left\{\sup _{y \in K}\|x-y\|\right\}$ is the Chebyshev radius of $K$ relative to itself. The space $E$ is said to have uniformly normal structure if $N(E)>1$. It is known that a uniformly convex Banach space has uniformly normal structure and for a Hilbert space $H, N(H)=\sqrt{2}$. Recently, Pichugov [18] (cf. Prus [20]) calculated that

$$
N\left(L^{p}\right)=\min \left\{2^{1 / p}, 2^{(p-1) / p}\right\}, \quad 1<p<\infty .
$$

Some estimates for normal structure coefficients in other Banach spaces may be found in [21].

For a subset $K$, we denote by $\overline{c o} K$ the closure of the convexity hull of $K$.

3. Main results. Now we are in position to give our result.

THEOREM 3.1. Let $p>1$ and let $E$ be a $p$-uniformly convex Banach space, $K a$ nonempty subset of $E, G$ a left reversible semitopological semigroup, and $\mathscr{Y}=\left\{T_{t}: t \in\right.$ $G$ \} a generalized Lipschitzian semigroup on $K$ with

$$
\left[\frac{(\alpha+\beta)^{p}\left(\alpha^{p} \cdot 2^{p-1}-1\right)}{\left(c_{p}-2^{p-1} \beta^{p}\right) \cdot N^{p}}\right]^{1 / p}<1,
$$

where

$$
\alpha=\limsup _{s} \frac{a_{s}+b_{s}+c_{s}}{1-b_{s}-c_{s}}, \quad \beta=\limsup _{s} \frac{2 b_{s}+2 c_{s}}{1-b_{s}-c_{s}} .
$$

Suppose that $\left\{T_{t} y: t \in G\right\}$ is bounded for some $y \in K$ and there exists a closed subset $C$ of $K$ such that $\bigcap_{s} \overline{\mathrm{CO}}\left\{T_{t} x: t \geq s\right\} \subseteq C$ for all $x \in K$. Then there exists a $z \in C$ such that $T_{s} z=z$ for all $s \in G$.

Proof. Let $B_{s}(x)=\overline{\operatorname{co}}\left\{T_{t} x: t \geq s\right\}$ and let $B(x)=\bigcap_{s} B_{s}(x)$ for $s \in G$ and $x \in K$. Define $\left\{x_{n}: n \geq 0\right\}$ by induction as follows:

$$
x_{0}=y, \quad x_{n}=A\left(\left\{B_{s}\left(x_{n-1}\right)\right\}, B\left(x_{n-1}\right)\right), \quad \text { for } n \geq 1 .
$$


Since $B(x) \subseteq C \subseteq K$ for all $x \in K,\left\{x_{n}\right\}$ is well defined. Let

$$
\begin{aligned}
r_{m} & =r\left(\left\{B_{s}\left(x_{m}\right)\right\}, B\left(x_{m}\right)\right), \\
D_{m} & =r\left(\left\{B_{s}\left(x_{m}\right)\right\}, B\left(x_{m-1}\right)\right), \quad m \geq 1 .
\end{aligned}
$$

Now, for each $s, t \in G$ and $x, y \in K$, we have

$$
\begin{aligned}
\left\|T_{s} T_{t} x-T_{s} y\right\| \leq & a_{s}\left\|T_{t} x-y\right\|+b_{s}\left(\left\|T_{t} x-T_{s} T_{t} x\right\|+\left\|y-T_{s} y\right\|\right) \\
& +c_{s}\left(\left\|y-T_{s} T_{t} x\right\|+\left\|T_{t} x-T_{s} y\right\|\right),
\end{aligned}
$$

and so

$$
\left\|T_{s} T_{t} x-T_{s} y\right\| \leq \frac{a_{s}+b_{s}+c_{s}}{1-b_{s}-c_{s}} \cdot\left\|T_{t} x-y\right\|+\frac{2 b_{s}+2 c_{s}}{1-b_{s}-c_{s}} \cdot\left\|y-T_{s} y\right\|
$$

Then from $x_{m} \in B\left(x_{m-1}\right)=\bigcap_{t} B_{t}\left(x_{m-1}\right)$ and a result of Ishihara and Takahashi [11], we have

$$
r_{m}=r\left(\left\{B_{s}\left(x_{m}\right)\right\}, B\left(x_{m}\right)\right) \leq \frac{1}{N} \cdot \inf _{s} \operatorname{diam}\left(B_{s}\left(x_{m}\right)\right)
$$

and by using (3.6), we have

$$
\begin{aligned}
\inf _{s} \operatorname{diam}\left(B_{s}\left(x_{m}\right)\right) & =\inf _{s} \sup \left\{\left\|T_{a} x_{m}-T_{b} x_{m}\right\|: a, b \geq s\right\} \\
& \leq \limsup _{t}\left(\limsup _{s}\left\|T_{s} x_{m}-T_{t} x_{m}\right\|\right) \\
& \leq \limsup _{t}\left(\limsup _{s}\left\|T_{t} T_{s} x_{m}-T_{t} x_{m}\right\|\right) \\
& \leq \limsup _{t}\left[\operatorname { l i m s u p } _ { s } \left\{\left(\frac{a_{t}+b_{t}+c_{t}}{1-b_{t}-c_{t}}\right) \cdot\left\|T_{s} x_{m}-x_{m}\right\|\right.\right. \\
& \left.\left.\quad+\left(\frac{2 b_{t}+2 c_{t}}{1-b_{t}-c_{t}}\right) \cdot\left\|x_{m}-T_{t} x_{m}\right\|\right\}\right] \\
& \leq(\alpha+\beta) \cdot D_{m},
\end{aligned}
$$

and hence

$$
r_{m} \leq \frac{\alpha+\beta}{N} \cdot D_{m}
$$

where $N$ is the normal structure coefficient of $E$. Again from (2.4) and (3.6) we have

$$
\begin{aligned}
& \left\|\lambda x_{m+1}+(1-\lambda) T_{t} x_{m+1}-T_{s} x_{m}\right\|^{p}+c_{p} \cdot W_{p}(\lambda) \cdot\left\|x_{m+1}-T_{t} x_{m+1}\right\|^{p} \\
& \leq \lambda\left\|x_{m+1}-T_{s} x_{m}\right\|^{p}+(1-\lambda) \cdot\left\|T_{t} x_{m+1}-T_{s} x_{m}\right\|^{p} \\
& \leq \lambda\left\|x_{m+1}-T_{s} x_{m}\right\|^{p}+(1-\lambda) \cdot\left\|T_{t} x_{m+1}-T_{t} T_{s} x_{m}\right\|^{p} \\
& \leq \lambda\left\|x_{m+1}-T_{s} x_{m}\right\|^{p}+(1-\lambda) \cdot\left[\frac{a_{t}+b_{t}+c_{t}}{1-b_{t}-c_{t}} \cdot\left\|T_{s} x_{m}-x_{m+1}\right\|\right. \\
& \left.+\frac{2 b_{t}+2 c_{t}}{1-b_{t}-c_{t}} \cdot\left\|T_{t} x_{m+1}-x_{m+1}\right\|\right]^{p} .
\end{aligned}
$$


Taking the limsup $\sup _{s}$, we have

$$
\begin{aligned}
r_{m}^{p}+ & c_{p} \cdot W_{p}(\lambda) \cdot\left\|x_{m+1}-T_{t} x_{m+1}\right\|^{p} \\
& \leq \lambda r_{m}^{p}+(1-\lambda)\left[\frac{a_{t}+b_{t}+c_{t}}{1-b_{t}-c_{t}} \cdot r_{m}+\frac{2 b_{t}+2 c_{t}}{1-b_{t}-c_{t}} \cdot\left\|T_{t} x_{m+1}-x_{m+1}\right\|\right]^{p} .
\end{aligned}
$$

It then follows that

$$
r_{m}^{p}+c_{p} \cdot W_{p}(\lambda) \cdot D_{m+1}^{p} \leq \lambda r_{m}^{p}+(1-\lambda) \cdot 2^{p-1}\left[\alpha^{p} r_{m}^{p}+\beta^{p} \cdot D_{m+1}^{p}\right]
$$

and so

$$
\begin{aligned}
D_{m+1}^{p} & \leq\left[\frac{(1-\lambda) \cdot\left(2^{p-1} \cdot \alpha^{p}-1\right)}{c_{p} \cdot W_{p}(\lambda)-(1-\lambda) \cdot 2^{p-1} \cdot \beta^{p}}\right] \cdot r_{m}^{p} \\
& \leq\left[\frac{(1-\lambda) \cdot\left(2^{p-1} \cdot \alpha^{p}-1\right)}{c_{p} \cdot W_{p}(\lambda)-(1-\lambda) \cdot 2^{p-1} \cdot \beta^{p}}\right] \cdot \frac{(\alpha+\beta)^{p}}{N^{p}} \cdot D_{m}^{p} .
\end{aligned}
$$

Letting $\lambda \rightarrow 1$, we conclude that

$$
D_{m+1} \leq\left[\frac{(\alpha+\beta)^{p}\left(2^{p-1} \cdot \alpha^{p}-1\right)}{\left(c_{p}-2^{p-1} \cdot \beta^{p}\right) \cdot N^{p}}\right]^{1 / p} \cdot D_{m}=A \cdot D_{m}, \quad m \geq 1,
$$

where

$$
A=\left[\frac{(\alpha+\beta)^{p}\left(2^{p-1} \cdot \alpha^{p}-1\right)}{\left(c_{p}-2^{p-1} \cdot \beta^{p}\right) \cdot N^{p}}\right]^{1 / p}<1
$$

by the assumption of the theorem. Since

$$
\begin{aligned}
\left\|x_{m+1}-x_{m}\right\| & \leq r\left(\left\{B_{s}\left(x_{m}\right)\right\}, x_{m+1}\right)+r\left(\left\{B_{s}\left(x_{m}\right)\right\}, x_{m}\right) \\
& \leq r_{m}+D_{m} \\
& \leq 2 D_{m} \\
& \vdots \\
& \leq 2 \cdot A^{m-1} D_{1} \longrightarrow 0 \quad \text { as } m \rightarrow \infty,
\end{aligned}
$$

it follows that $\left\{z_{m}\right\}$ is a Cauchy sequence. Let $z=\lim _{m \rightarrow \infty} x_{m}$. Then we have

$$
\begin{aligned}
\left\|z-T_{s} z\right\| \leq & \left\|z-x_{m}\right\|+\left\|x_{m}-T_{s} x_{m}\right\|+\left\|T_{s} x_{m}-T_{s} z\right\| \\
\leq & \left\|z-x_{m}\right\|+\left\|x_{m}-T_{s} x_{m}\right\|+\frac{a_{s}+b_{s}+c_{s}}{1-b_{s}-c_{s}}\left\|z-x_{m}\right\| \\
& +\frac{2 b_{s}+2 c_{s}}{1-b_{s}-c_{s}}\left\|x_{m}-T_{s} x_{m}\right\| \\
\leq & \frac{1+a_{s}}{1-b_{s}-c_{s}} \cdot\left\|z-x_{m}\right\|+\frac{1+b_{s}+c_{s}}{1-b_{s}-c_{s}} \cdot\left\|x_{m}-T_{s} x_{m}\right\| .
\end{aligned}
$$


Taking the limit as $m \rightarrow \infty$ on each side, we have

$$
\left\|z-T_{s} z\right\| \leq \lim _{m \rightarrow \infty}\left[\frac{1+a_{s}}{1-b_{s}-c_{s}} \cdot\left\|z-x_{m}\right\|+\frac{1+b_{s}+c_{s}}{1-b_{s}-c_{s}} \cdot D_{m}\right]=0
$$

for all $s \in G$. Hence we have $T_{s} z=z$ for all $s \in G$. This completes the proof.

REMARK 3.2. Theorem 3.1 is also true for Lipschitzian semigroup $\mathscr{S}=\left\{T_{t}: t \in G\right\}$ on $K$ with

$$
\limsup _{s} k_{s}<\left[\frac{1}{2}\left(1+\sqrt{1+4 \cdot c_{p} \cdot N^{p}}\right)\right]^{1 / p} .
$$

As a direct consequence of Theorem 3.1, we have the following result.

COROLlary 3.3. Let $p>1$ and let $E$ be a $p$-uniformly convex Banach space, $K a$ nonempty subset of $E$, and $T$ a mapping from $K$ into itself such that

$$
\begin{aligned}
\left\|T^{n} x-T^{n} y\right\| \leq & a_{n}\|x-y\|+b_{n}\left(\left\|x-T^{n} x\right\|+\left\|y-T^{n} y\right\|\right) \\
& +c_{n}\left(\left\|x-T^{n} y\right\|+\left\|y-T^{n} x\right\|\right),
\end{aligned}
$$

for each $x, y \in K$ and $n \geq 1$, where $a_{n}, b_{n}, c_{n}$ are the nonnegative constants such that there exists an integer $n_{0}$ such that $b_{n}+c_{n}<1$ for all $n \geq n_{0}$. Suppose that $\left\{T^{n} y: n \geq 1\right\}$ is bounded for some $y \in K$ and there exists a closed subset $C$ of $K$ such that $\bigcap_{n} \overline{\operatorname{co}}\left\{T^{n} x: k \geq n\right\} \subseteq C$ for all $x \in K$. If

$$
\left[\frac{(\alpha+\beta)^{p}\left(\alpha^{p} \cdot 2^{p-1}-1\right)}{\left(c_{p}-2^{p-1} \beta^{p}\right) \cdot N^{p}}\right]^{1 / p}<1,
$$

where

$$
\alpha=\limsup _{n} \frac{a_{n}+b_{n}+c_{n}}{1-b_{n}-c_{n}}, \quad \beta=\limsup _{n} \frac{2 b_{n}+2 c_{n}}{1-b_{n}-c_{n}},
$$

then there exists a $z \in C$ such that $T z=z$.

4. Some applications. In a Hilbert space $H$, the following equality holds:

$$
\|\lambda x+(1-\lambda) y\|^{2}=\lambda\|x\|^{2}+(1-\lambda)\|y\|^{2}-\lambda(1-\lambda)\|x-y\|^{2}
$$

for all $x, y$ in $H$ and $\lambda \in[0,1]$.

By Theorem 3.1 and (4.1), we immediately obtain the following.

THEOREM 4.1. Let $K$ be a nonempty subset of a Hilbert space $H, G$ a left reversible semitopological semigroup, and $\mathscr{S}=\left\{T_{t}: t \in G\right\}$ a generalized Lipschitzian semigroup on $K$ with

$$
\left[\frac{(\alpha+\beta)^{2}\left(2 \alpha^{2}-1\right)}{2\left(1-2 \beta^{2}\right)}\right]^{1 / 2}<1,
$$

where $\alpha, \beta$ are as in Theorem 3.1. Suppose that $\left\{T_{t} y: t \in G\right\}$ is bounded for some $y \in K$ and there exists a closed subset $C$ of $K$ such that $\bigcap_{s} \overline{\mathrm{co}}\left\{T_{t} x: t \geq s\right\} \subseteq C$ for all $x \in K$. Then there exists $z \in C$ such that $T_{s} z=z$ for all $s \in G$. 
The following result follows easily from Theorem 4.1.

COROLLARY 4.2 (see [10, Theorem 1]). Let $K$ be a nonempty subset of a Hilbert space $H, G$ a left reversible semitopological semigroup, and $\mathscr{Y}=\left\{T_{t}: t \in G\right\}$ a Lipschitzian semigroup on $K$ with $\lim \sup _{s} k_{s}<\sqrt{2}$. Suppose that $\left\{T_{t} y: t \in G\right\}$ is bounded for some $y \in K$ and there exists a closed subset $C$ of $K$ such that $\bigcap_{s} \overline{\operatorname{co}}\left\{T_{t} x: t \geq s\right\} \subseteq C$ for all $x \in K$. Then there exists $z \in C$ such that $T_{s} z=z$ for all $s \in G$.

If $1<p \leq 2$, then we have for all $x, y$ in $L^{p}$ and $\lambda \in[0,1]$,

$$
\|\lambda x+(1-\lambda) y\|^{2} \leq \lambda\|x\|^{2}+(1-\lambda)\|y\|^{2}-\lambda(1-\lambda)(p-1)\|x-y\|^{2} .
$$

(The inequality (4.3) is contained in [16, 23].)

Assume that $2<p<\infty$ and $t_{p}$ is the unique zero of the function $g(x)=-x^{p-1}+$ $(p-1) x+p-2$ in the interval $(1, \infty)$. Let

$$
c_{p}=(p-1)\left(1+t_{p}\right)^{2-p}=\frac{1+t_{p}^{p-1}}{\left(1+t_{p}\right)^{p-1}} .
$$

Then we have the following inequality:

$$
\|\lambda x+(1-\lambda) y\|^{p} \leq \lambda\|x\|^{p}+(1-\lambda)\|y\|^{p}-W_{p}(\lambda) \cdot c_{p} \cdot\|x-y\|^{p}
$$

for all $x, y$ in $L^{p}$ and $\lambda \in[0,1]$. (Inequality (4.5) is essentially due to Lim [15].)

By inequality (4.3) and (4.5), we immediately obtain from Theorem 3.1 the following result.

THEOREM 4.3. Let $K$ be a nonempty subset of $L^{p}, 1<p<\infty, G$ a left reversible semitopological semigroup, and $\mathscr{Y}=\left\{T_{t}: t \in G\right\}$ a generalized Lipschitzian semigroup on $K$ with

$$
\begin{aligned}
& {\left[\frac{(\alpha+\beta)^{2}\left(2 \alpha^{2}-1\right)}{2^{(p-1) / p}\left(p-1-2 \beta^{2}\right)}\right]^{1 / 2}<1 \quad \text { for } 1<p \leq 2,} \\
& {\left[\frac{(\alpha+\beta)^{p} \cdot\left(2^{p-1} \alpha^{p}-1\right)}{\left(c_{p}-2^{p-1} \beta^{p}\right) \cdot 2}\right]^{1 / p}<1 \quad \text { for } 2<p<\infty,}
\end{aligned}
$$

where $\alpha, \beta$ are as in Theorem 2.4. Suppose that $\left\{T_{t} y: t \in G\right\}$ is bounded for some $y \in K$ and there exists a closed subset $C$ of $K$ such that $\bigcap_{s} \overline{\operatorname{co}}\left\{T_{t} x: t \succeq s\right\} \subseteq C$ for all $x \in K$. Then there exists $z \in C$ such that $T_{s} z=z$ for all $s \in G$.

REMARK 4.4. Theorem 4.1 is also true for Lipschitzian semigroup $\mathscr{S}=\left\{T_{t}: t \in G\right\}$ on $K$ with

$$
\begin{aligned}
& \limsup _{s} k_{s}<\left[\frac{1}{2}\left(1+\sqrt{1+4 \cdot(p-1) \cdot 2^{(p-1) / p}}\right)\right]^{1 / p} \text { for } 1<p \leq 2, \\
& \limsup _{s} k_{s}<\left[\frac{1}{2}\left(1+\sqrt{1+8 \cdot c_{p}}\right)\right]^{1 / p} \text { for } 2<p<\infty
\end{aligned}
$$


Let $H^{p}, 1<p<\infty$, denote the Hardy space [6] of all functions $x$ analytic in unit disc $|z|<1$ of the complex plane and such that

$$
\|x\|=\lim _{r \rightarrow 1^{-}}\left(\frac{1}{2 \pi} \int_{0}^{2 \pi}\left|x\left(r e^{i \theta}\right)\right|^{p} d \theta\right)^{1 / p}<\infty .
$$

Now, let $\Omega$ be an open subset of $\mathbb{R}^{n}$. Denote by $H^{k, p}(\Omega), k \geq 0,1<p<\infty$, the Sobolev space [1, page 149] of distributions $x$ such that $D^{\alpha} x \in L^{p}(\Omega)$ for all $|\alpha|=$ $\alpha_{1}+\cdots+\alpha_{n} \leq k$ equipped with the norm

$$
\|x\|=\left(\sum_{|\alpha| \leq k} \int_{\Omega}\left|D^{\alpha} x(\omega)\right|^{p} d \omega\right)^{1 / p} .
$$

Let $\left(\Omega_{\alpha}, \sum_{\alpha}, \mu_{\alpha}\right), \alpha \in \Lambda$, be a sequence of positive measure spaces, where the index set $\Lambda$ is finite or countable. Given a sequence of linear subspaces $X_{\alpha}$ in $L^{p}\left(\Omega_{\alpha}, \sum_{\alpha}, \mu_{\alpha}\right)$, we denote by $L_{q, p}, 1<p<\infty$ and $q=\max \{2, p\}$ (see [17]), the linear space of all sequences $x=\left\{x_{\alpha} \in X_{\alpha}: \alpha \in \Lambda\right\}$ equipped with the norm

$$
\|x\|=\left(\sum_{\alpha \in \Lambda}\left(\left\|x_{\alpha}\right\|_{p, \alpha}\right)^{q}\right)^{1 / q}
$$

where $\|\cdot\|_{p, \alpha}$ denotes the norm in $L^{p}\left(\Omega_{\alpha}, \sum_{\alpha}, \mu_{\alpha}\right)$.

Finally, let $L_{p}=L^{p}\left(S_{1}, \sum_{1}, \mu_{1}\right)$ and $L_{q}=L^{q}\left(S_{2}, \sum_{2}, \mu_{2}\right)$, where $1<p<\infty, q=$ $\max \{2, p\}$ and $\left(S_{i}, \sum_{i}, \mu_{i}\right)$ are positive measure spaces. Denote by $L_{q}\left(L_{p}\right)$ the Banach spaces [5, Chapter III, Section 2, Definition 10] of all measurable $L_{p}$-value function $x$ on $S_{2}$ such that

$$
\|x\|=\left(\int_{S_{2}}\left(\|x(s)\|_{p}\right)^{q} \mu_{2}(d s)\right)^{1 / q} .
$$

These spaces are $q$-uniformly convex with $q=\max \{2, p\}$ (see $[19,22]$ ), and the norm in these spaces satisfies

$$
\|\lambda x+(1-\lambda) y\|^{q} \leq \lambda\|x\|^{q}+(1-\lambda)\|y\|^{q}-d \cdot W_{q}(\lambda) \cdot\|x-y\|^{q}
$$

with a constant

$$
d=d_{p}= \begin{cases}\frac{p-1}{8} & \text { for } 1<p \leq 2 \\ \frac{1}{p \cdot 2^{p}} & \text { for } 2<p<\infty\end{cases}
$$

Now from Theorem 3.1, we have the following result.

THEOREM 4.5. Let $K$ be a nonempty subset of the space $E$, where $E=H^{p}$, or $E=$ $H^{k, p}(\Omega)$, or $E=L_{q, p}$, or $E=L_{q}\left(L_{p}\right)$, and $1<p<\infty, q=\max \{2, p\}, k \geq 0$. Let $G$ be a left reversible semitopological semigroup and $\mathscr{S}=\left\{T_{t}: t \in G\right\}$ a generalized Lipschitzian semigroup on $K$ with

$$
\left[\frac{(\alpha+\beta)^{q}\left(\alpha^{q} \cdot 2^{q-1}-1\right)}{\left(d-2^{q-1} \beta^{q}\right) \cdot N^{q}}\right]^{1 / q}<1,
$$


where $\alpha, \beta$ are as in Theorem 3.1. Suppose that $\left\{T_{t} y: t \in G\right\}$ is bounded for some $y \in K$ and there exists a closed subset $C$ of $K$ such that $\bigcap_{s} \overline{\operatorname{co}}\left\{T_{t} x: t \geq s\right\} \subseteq C$ for all $x \in K$. Then there exists $z \in C$ such that $T_{s} z=z$ for all $s \in G$.

REMARK 4.6. Theorem 4.5 is also true for Lipschitzian semigroup $\mathscr{S}=\left\{T_{t} y: t \in G\right\}$ on $K$ with

$$
\limsup _{s} k_{s}<\left[\frac{1}{2}\left(1+\sqrt{1+4 \cdot d \cdot N^{q}}\right)\right]^{1 / q} .
$$

ACKNOWLEDGEMENT. This work was supported by Korea Research Foundation Grant (KRF-2000-DP0013).

\section{REFERENCES}

[1] J. Barros-Neto, An Introduction to the Theory of Distributions, Pure and Applied Mathematics, vol. 14, Marcel Dekker, New York, 1973. MR 57\#1113. Zbl 273.46026.

[2] W. L. Bynum, Normal structure coefficients for Banach spaces, Pacific J. Math. 86 (1980), no. 2, 427-436. MR 81m:46030. Zbl 442.46018.

[3] E. Casini and E. Maluta, Fixed points of uniformly Lipschitzian mappings in spaces with uniformly normal structure, Nonlinear Anal. 9 (1985), no. 1, 103-108. MR 86m:47082. Zbl 553.47021.

[4] D. J. Downing and W. O. Ray, Uniformly Lipschitzian semigroups in Hilbert space, Canad. Math. Bull. 25 (1982), no. 2, 210-214. MR 84e:47066. Zbl 475.47045.

[5] N. Dunford and J. T. Schwartz, Linear Operators. I. General Theory, Pure and Applied Mathematics, vol. 7, Interscience Publishers, New York, 1958. MR $22 \# 8302$. Zbl 084.10402.

[6] P. L. Duren, Theory of $H^{p}$ Spaces, Academic Press, New York, 1970. MR $42 \# 3552$. Zbl 215.20203.

[7] K. Goebel and W. A. Kirk, A fixed point theorem for transformations whose iterates have uniform Lipschitz constant, Studia Math. 47 (1973), 135-140. MR 49\#1242. Zbl 265.47044.

[8] _ _ Topics in Metric Fixed Point Theory, Cambridge Studies in Advanced Mathematics, vol. 28, Cambridge University Press, Cambridge, 1990. MR 92c:47070. Zbl 708.47031.

[9] K. Goebel, W. A. Kirk, and R. L. Thele, Uniformly Lipschitzian families of transformations in Banach spaces, Canad. J. Math. 26 (1974), 1245-1256. MR 50\#10919. Zbl 285.47039.

[10] H. Ishihara, Fixed point theorems for Lipschitzian semigroups, Canad. Math. Bull. 32 (1989), no. 1, 90-97. MR 90f:47084. Zbl 638.47061.

[11] H. Ishihara and W. Takahashi, Fixed point theorems for uniformly Lipschitzian semigroups in Hilbert spaces, J. Math. Anal. Appl. 127 (1987), no. 1, 206-210. MR 88j:47076. Zbl 637.47028.

[12] _ Modulus of convexity, characteristic of convexity and fixed point theorems, Kodai Math. J. 10 (1987), no. 2, 197-208. MR 88f:46040.

[13] E. A. Lifšic, A fixed point theorem for operators in strongly convex spaces, Voronež. Gos. Univ. Trudy Mat. Fak. 16 (1975), 23-28. MR 57\#17401.

[14] T. C. Lim, On asymptotic centers and fixed points of nonexpansive mappings, Canad. J. Math. 32 (1980), no. 2, 421-430. MR 81k:47081. Zbl 454.47045.

[15] _ On some $L^{p}$ inequalities in best approximation theory, J. Math. Anal. Appl. 154 (1991), no. 2, 523-528. MR 92d:41029. Zbl 744.41015.

[16] T. C. Lim, H. K. Xu, and Z. B. Xu, Some L ${ }^{p}$ Inequalities and Their Applications to Fixed Point Theory and Approximation Theory, Progress in Approximation Theory, Academic Press, Massachusetts, 1991, pp. 609-624. MR 92j:47112. 
[17] J. Lindenstrauss and L. Tzafriri, Classical Banach Spaces. II. Function Spaces, Ergebnisse der Mathematik und ihrer Grenzgebiete [Results in Mathematics and Related Areas], vol. 97, Springer-Verlag, Berlin, 1979. MR 81c:46001. Zbl 403.46022.

[18] S. A. Pichugov, The Jung constant of the space $L_{p}$, Mat. Zametki 43 (1988), no. 5, 604-614, translation in Math. Notes 43 (1988), 348-354. MR 90a:46037. Zbl 663.46021.

[19] B. Prus and R. Smarzewski, Strongly unique best approximations and centers in uniformly convex spaces, J. Math. Anal. Appl. 121 (1987), no. 1, 10-21. MR 88i:41052. Zbl 617.41046.

[20] S. Prus, On Bynum's fixed point theorem, Atti Sem. Mat. Fis. Univ. Modena 38 (1990), no. 2, 535-545. MR 91k:47146. Zbl 724.46020.

[21]_ Some estimates for the normal structure coefficient in Banach spaces, Rend. Circ. Mat. Palermo (2) 40 (1991), no. 1, 128-135. MR 92j:46029. Zbl 757.46029.

[22] R. Smarzewski, Strongly unique best approximation in Banach spaces. II, J. Approx. Theory 51 (1987), no. 3, 202-217. MR 88j:41064. Zbl 657.41022.

[23] _ On an inequality of Bynum and Drew, J. Math. Anal. Appl. 150 (1990), no. 1, 146-150. MR 91g:47046. Zbl 716.46023.

[24] H. K. Xu, Inequalities in Banach spaces with applications, Nonlinear Anal. 16 (1991), no. 12, 1127-1138. MR 92e:47126. Zbl 757.46033.

[25] C. Zălinescu, On uniformly convex functions, J. Math. Anal. Appl. 95 (1983), no. 2, 344374. MR 85a:26018. Zbl 519.49010.

Jong Soo Jung: Department of Mathematics, Dong-A University, Pusan 607-714, KOREA

E-mail address: jungjs@mai 1.donga.ac.kr

BALWANT Singh ThaKUR: Govt. B. H. S. S. GARIABAND, Dist. RAIPUR (M. P.) 493889, INDIA 


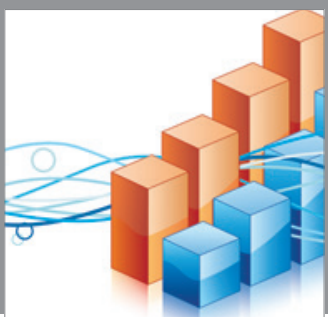

Advances in

Operations Research

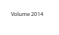

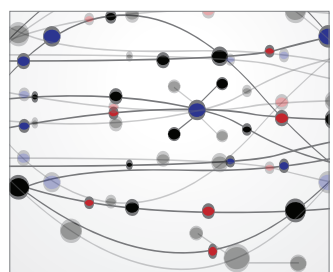

\section{The Scientific} World Journal
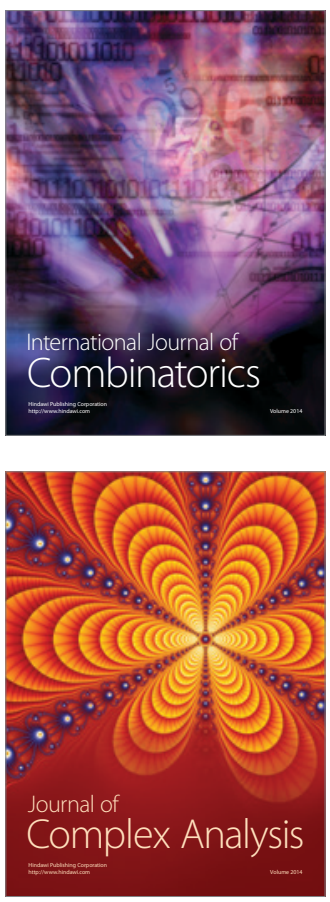

International Journal of

Mathematics and

Mathematical

Sciences
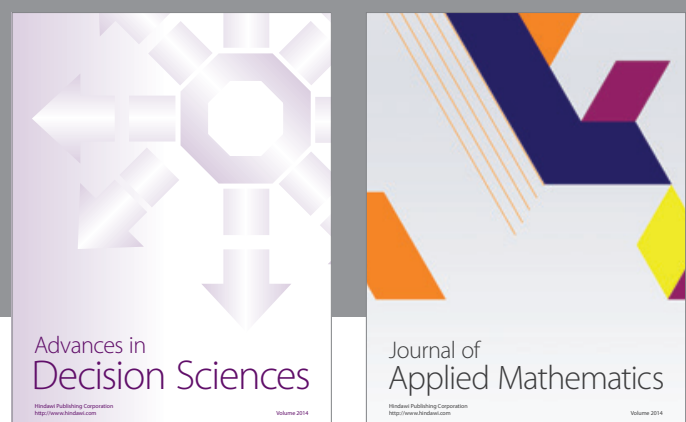

Journal of

Applied Mathematics
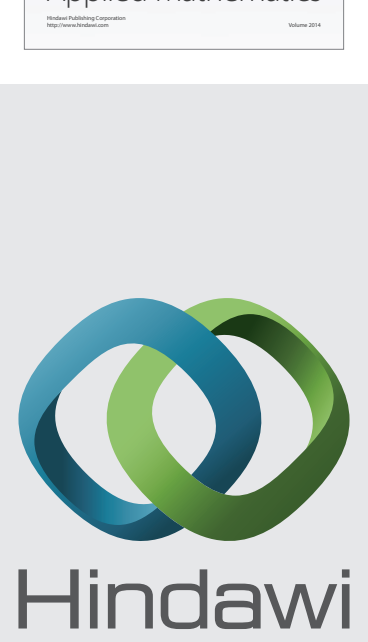

Submit your manuscripts at http://www.hindawi.com
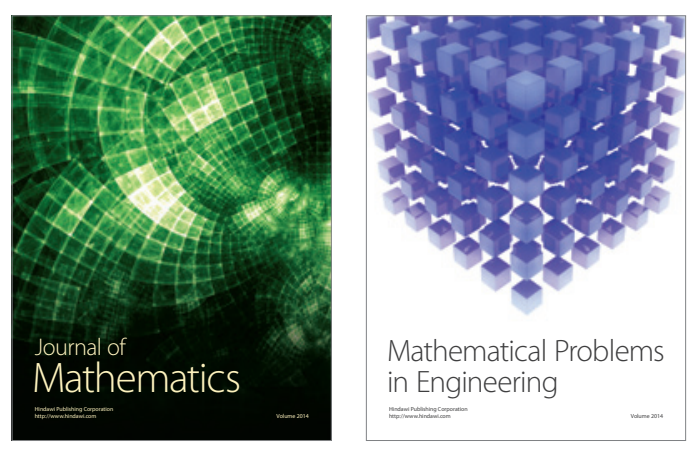

Mathematical Problems in Engineering
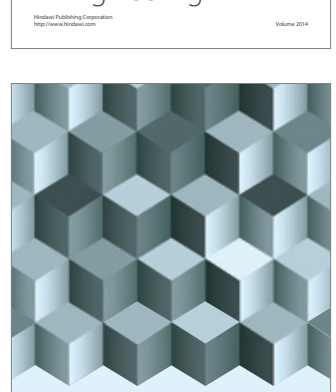

Journal of

Function Spaces
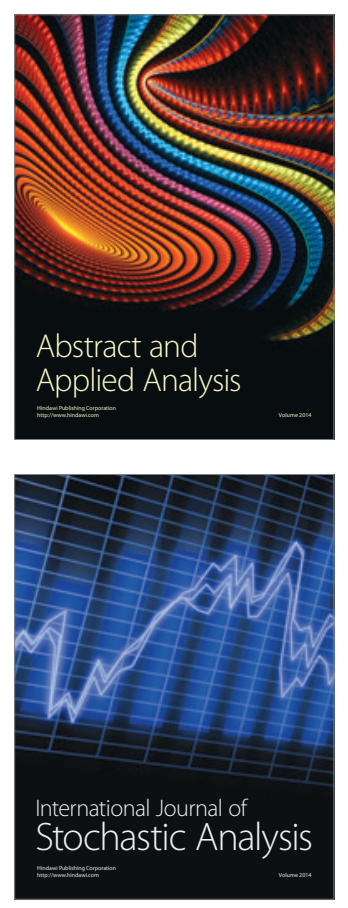

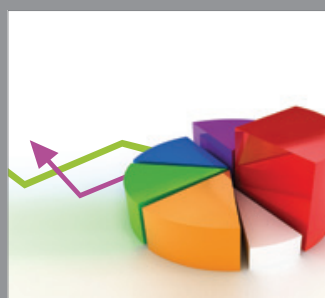

ournal of

Probability and Statistics

Promensencen
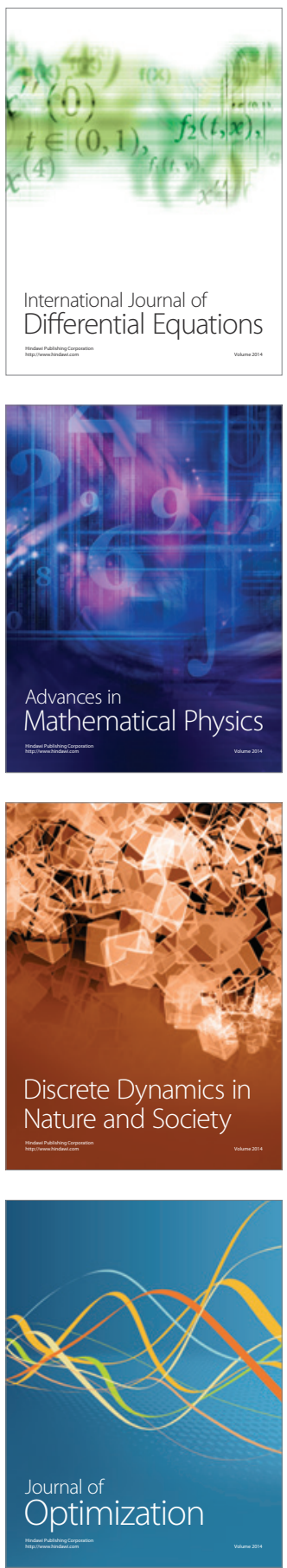